\title{
Erratum to: Synergistic effect of programmed cell death protein 1 blockade and secondary lymphoid tissue chemokine in the induction of anti-tumor immunity by a therapeutic cancer vaccine
}

\author{
Soheila Moeini ${ }^{1}$ - Mohsen Saeidi ${ }^{2}$ - Fatemeh Fotouhi ${ }^{3} \cdot$ Mahdieh Mondanizadeh $^{4}$. \\ Sadegh Shirian $^{5} \cdot$ Alireza Mohebi $^{6,7} \cdot$ Ali Gorji $^{8,9} \cdot$ Amir Ghaemi $^{3}$
}

Published online: 24 November 2016

(C) Springer-Verlag Wien 2016

\section{Erratum to: Arch Virol}

DOI 10.1007/s00705-016-3091-5

Unfortunately, the fourth author name "Mahdieh Mondanizadeh" was incorrectly published in the original version. The author name is corrected here and in the original publication as well.

The original article was corrected.

The online version of the original article can be found under doi:10.1007/s00705-016-3091-5.

Amir Ghaemi

ghaem_amir@yahoo.com; a_ghaemi@pasteur.ac.ir

1 Mashhad University of Medical Sciences, Mashhad, Iran

2 Stem Cell Research Center, Golestan University of Medical Sciences, Gorgan, Iran

3 Department of Virology, Pasteur Institute of Iran, P.O.Box: 1316943551, Tehran, Iran

4 Biotechnology and molecular medicine, Arak University of medical sciences, Arak, Iran

5 Department of Pathology, School of Veterinary Medicine, Shahrekord University, Shahrekord, Iran

6 Department of Microbiology, Golestan University of Medical Sciences, Gorgan, Iran

7 Infectious Diseases Research Center, Department of Microbiology, Golestan University of Medical Sciences, Gorgan, Iran

8 Institut für Physiologie I, Westfälische Wilhelms-Universität Münster, Robert-Koch-Strasse Münster, Germany

9 Shefa Neuroscience Research Center, Tehran, Iran 